\title{
Massive Bleeding After Median Sternotomy: Case Report of an Infrequent Complication of Infective Endocarditis
}

\author{
Laura Varela Barca, Jose Lopez Menendez, Ana Redondo Palacios, Jorge Rodriguez Roda \\ Stuart
}

University Hospital Ramón y Cajal, Madrid, Spain

Received: 01 May, 2017 ; Accepted: 25 May, 2017; Published: 05 June, 2017

*Corresponding author: Laura Varela Barca, University Hospital Ramón y Cajal, Madrid, Spain, Tel no: (+34) 617103918; E-mail: lauravarela21089@gmail.com

\begin{abstract}
We report an unusual complication of acute infective endocarditis, found in a 70 year-old man with a previous history of two cardiac surgery procedures. The first one was a full root replacement (BentallDe Bono procedure), associated with three bypasses (one mammary and two vein grafts). The second procedure was a mitral valve replacement through a right thoracotomy ought to native mitral valve endocarditis.
\end{abstract}

In the current admission, echocardiography showed a periaortic abscess (Figure 1) and severe prosthetic mitral valve insufficiency. CT scan showed a big collection in the anterior mediastinal space adjacent to the composite graft (Figure 2), which was suspected to be purulent material.

A third surgical intervention was programmed despite the extreme surgical risk. During the median sternotomy, a massive bleeding occurred. It was originated in the mediastinal collection, which truly was a collection of contained bleeding, originated in the 10 yearsold anastomosis of the saphenous vein to the composite graft, which was completely detached due to endocarditic involvement of the aortic graft. The institution of cardiopulmonary bypass before sternal opening enabled the surgical team to maintain a stable hemodynamic situation and to continue the procedure satisfactorily.

Keywords: Infectious endocarditis; Composite graft; Combined surgery

\section{Introduction}

Infective endocarditis (IE) is an important cause of cardiovascular pathology that, despite diagnostic and therapeutic advances, it continues to be a serious disease that leads to a high morbidity and mortality [1]. The presence of local complications, (such as periannular tissue destruction, abscess...) increases the complexity and mortality associated with IE surgery [2]. An episode of IE affecting a composite graft after aortic root replacement can be a life-threatening complication $[3,4]$. Moreover, redo procedures after a previous CAGB surgery, with a patent internal mammary artery (IMA), may significantly increase the risk of the surgical procedure [5].
We report the case of 70-year-old man with an episode of IE affecting a composite aortic root and mitral prosthesis, who had an extremely high surgical risk, in whom a previous saphenous vein graft was found to be detached from the aortic graft because of endocarditic involvement, forming a huge collection of blood in the anterior mediastinum.

\section{Case Report}

A 70 year-old man was emergently referred to the Cardiac Surgery Department due to a confirmed diagnosis of acute IE. The patient had a previous clinical history of arterial hypertension and atrial fibrillation. He had two previous cardiac surgery procedures. He had undergone, 10 years ago, a full aortic root replacement with a composite graft (Dacron graft with mechanical valve, Bentall-De Bono procedure) associated with triple coronary bypass (IMA to anterior descendent artery, and vein graft to intermediate branch and posterior descendent artery) [6].

The second procedure was six months before the current episode, when he suffered a native mitral valve IE episode (caused by S. Epidermidis). He was admitted in cardiogenic shock with confirmed endocarditic involvement of the mitral valve. The critical preoperative status did not permit to make a preoperative angiogram. No signs of myocardial ischemia were present, so he had an emergent mitral valve replacement by a mechanical prosthesis performed through a right thoracotomy approach, to avoid a possible complication related to previous coronary grafts. The postoperative course was uneventful, and the patient was discharged home after completing six weeks of intravenous antibiotic treatment with daptomycin and rifampicin.

The current episode started when the patient was readmitted to our Institution in a critical clinical state, with congestive heart failure, and a septic status. The patient presented persistent fever, dyspnea, orthopnea and paroxysmal nocturnal dyspnea. In the physical examination, no peripheral stigmata of endocarditis were found. A diastolic murmur was heard along the left sternal border. Three blood cultures were positive to 
S. aureus. Antibiotic treatment was initiated with intravenous oxacilin, rifampicin and gentamicin. Complete imaging studies were conducted.

The transesophageal echocardiography revealed a mitral peri-prosthetic leak that caused severe mitral valve regurgitation. There were vegetations in both aortic and mitral prosthetic valves. Annular affectation was diagnosed, with the presence of a large perivalvular aortic abscess (Figure 1).

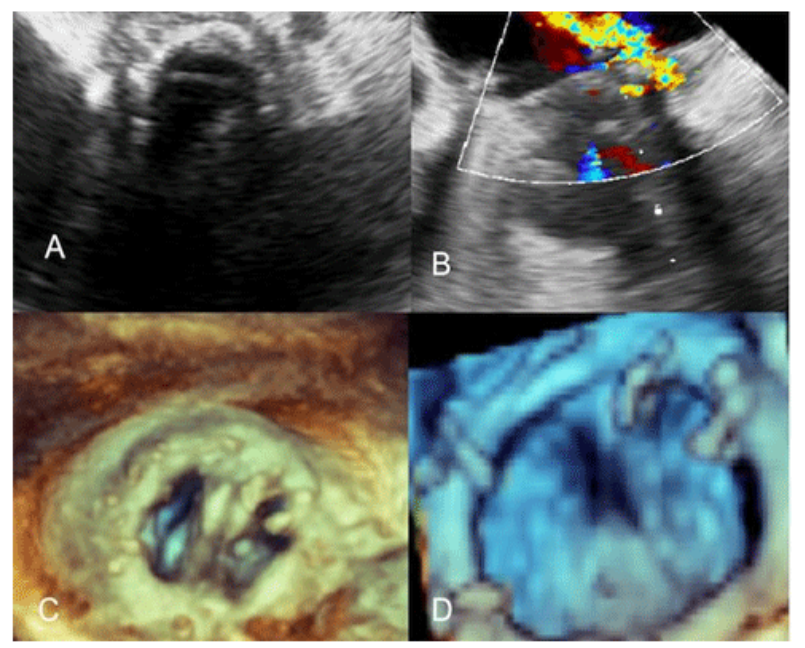

Figure 1: Transesophageal echocardiography. (A). Prosthetic aortic valve with a large perivalvular aortic abscess. (B). Mitral peri-prosthetic leak that caused severe mitral valve regurgitation. (C): 3D Reconstruction of mitral valve. (D): Vegetations in mitral prosthetic valve.

The coronariography showed atherosclerotic coronary disease of the anterior descendent and distal circumflex arteries. All the previous bypasses were not patent. It was not possible to selectively make an injection in the previous vein grafts. The comparation between the coronariography with the previous one was impossible, no coronariography was performed in the previous mitral IE surgery due to the urgency of the intervention.

A preoperative thoracic CT scan was performed, and it showed a huge collection of unknown origin in the anterior mediastinal space, of $100 \times 55 \times 75 \mathrm{~mm}$, adjacent to the Dacron composite graft, in its anterior aspect, and in close contact with the thoracic wall (Figure 2). It was a clearly delimited collection, with dense and heterogeneous content. As there were no images of flow of contrast inside this collection, it was suspected to be purulent material. Soft tissue attenuation is described around the insertion of the mechanical aortic valve, suggestive of active IE. After a short period of stabilization and antibiotic therapy of 13 days, the patient underwent surgery.

Before opening the sternum, due to the suspected high risk of rupture, arterial cannulation was performed in the axillary artery and femoral vein, cardiopulmonary bypass and hypothermia were established. During the sternal opening, a massive acute bleeding occurred. The bleeding was partially controlled by the occlusion of the bleeding point by digital pressure through the partially opened sternum. After completing the sternal opening, the site of bleeding was identified. It was caused by the complete detachment from the aorta of the previous vein graft anastomosis, ought to endocarditic affectation. The institution of cardiopulmonary bypass before sternal opening allowed to maintain a stable hemodynamic situation, and the bleeding site could be controlled.

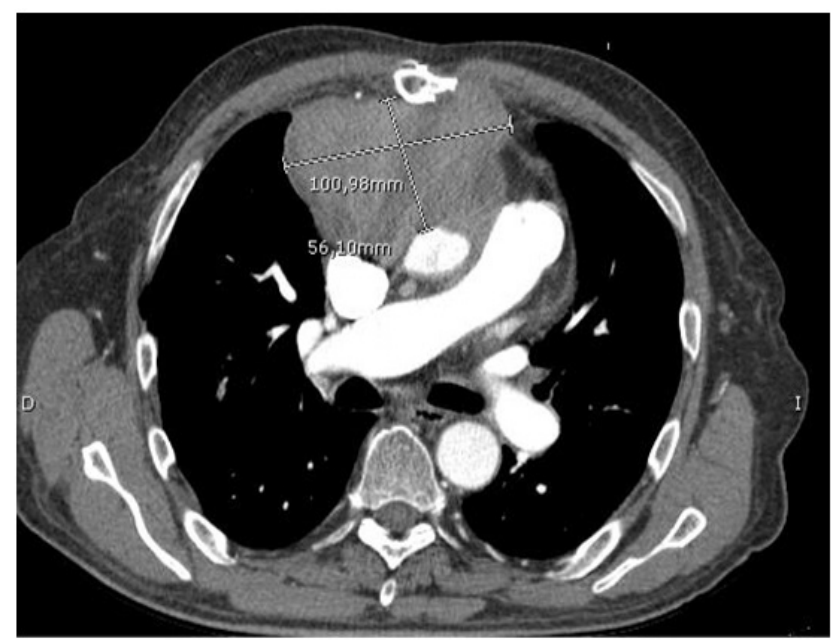

Figure 2: Thoracic TC scan with intravenous contrast. Axial section showing a clearly delimited collection in the mediastinal space adjacent to the Dacron composite graft.

All the affected tissues were excised, including the debridement of all infected and necrotic regions. The patient underwent a mitral valve replacement and a full root replacement with a mechanical composite graft (Medtronic Inc., Minneapolis, MN). It was not possible to mobilize the native coronary ostia because of the firm adhesions due to the previous surgeries and the large IE affectation, so the new Bentall-De Bono procedure was performed with the Cabrol modification with an $8 \mathrm{~mm}$ Dacron graft [7]. As there was no suspected ischemic heart disease in the preoperative checkup, no new coronary bypasses were constructed. Despite the prolonged aortic cross clamp, the myocardial ischemia was 206 minutes and the pump time was 300 minutes, no temporary circulatory support was necessary. The patient was transferred to the Intense Care Unit in a stable hemodynamic situation, with low dose inotropic drugs and an optimal hemodynamic state. Unfortunately, in the second postoperative day, the patient suffered an episode of septic shock and he died due to an acute septic shock refractory to medical treatment. The cause of the septic shock was a bacteriemia due to Klebsiella pneumoniae (Carbapenemases producer). There was no insolation of S. aureus in the postoperative blood cultures. 


\section{Discussion}

The presence of IE perivalvular complications increase the complexity associated with IE surgery (2). Periannular tissue destruction, abscess formation, prosthesis dehiscence, presence of vegetation and pseudoaneurism formation are serious complications associated with a high mortality rate [4]. In addition, composite graft IE after aortic root replacement can be a lifethreating complication, and recurrent valve IE remains a surgical challenge because a more radical and aggressive surgical treatment is required to prevent a recurrent infection $[3,8]$.

We report a case with an extremely high surgical risk, which was a second episode of IE, with an estimated 30-days mortality by EuroScore I and II of $81.6 \%$ and $42.6 \%$ respectively. The case was discussed in the multidisciplinary endocarditis team, with the collaboration of cardiologist, microbiologist, and cardiac surgeons, and despite the extreme surgical risk the decision to proceed with surgery was accepted, as it was the only chance for survival. Surgery appeared to be the only possible strategy based on the clinical management guides [1,9].

Moreover, we found a very infrequent complication of IE: the total detachment of a previous vein graft anastomosis ought to endocarditic involvement of the ascending aortic graft, with the formation of a big collection of blood contained by the adhesions of the previous surgeries. Some case reports were previously published showing the severity of cardiac involvement of IE. Prosthesis detachment due to IE affection and ischemia due to abscess compromise were described, but no literature was found of bypass graft affectation $[10,11]$.

Due to the absence of similar reports in the previously published literature, we consider that this case is a good example of an extreme IE affectation. Moreover, our finding may help the planning of the surgical strategy in the unusual case of a mediastinal collection in a patient with IE affecting a previous full-root graft and saphenous vein grafts. Cannulation for cardiopulmonary bypass before opening the sternum allowed the surgical team to control the potential fatal complication of massive bleeding. Periphereal cannulation prior to sternal opening could be mandatory in IE destructive cases, when the destruction of outer heart structures is suspected.

\section{References}

1. Habib G, Lancellotti P, Antunes MJ, Bongiorni MG, Casalta J-P, Zotti FD, et al. ESC Guidelines for the management of infective endocarditis. Eur Heart J. 2015;36(44):3075-3128. doi: 10.1093/eurheartj/ehv319

2. Lee S, Chang BC, Park HK. Surgical experience with infective endocarditis and aortic root abscess. Yonsei Med J. 2014;55(5):1253-1259. doi: 10.3349/ymj.2014.55.5.1253

3. Ramos A, García-Montero C, López-Menéndez J, Muñoz P, Ruiz-Morales J, Sánchez-Espín G, et al. Endocarditis in patients with ascending aortic prosthetic graft: a case series from a national multicentre registry. Eur J Cardiothorac Surg. 2016;50(6):1149-1157. doi: 10.1093/ejcts/ ezw190

4. Apaydin AZ, Posacioglu H, Islamoglu F, Degirmenciler K, Durmaz I. Composite Graft Endocarditis. Tex Heart Inst J. 2004;31(3):306-308.

5. Byrne JG, Karavas AN, Filsoufi F, Mihaljevic T, Aklog L, Adams DH, et al. Aortic valve surgery after previous coronary artery bypass grafting with functioning internal mammary artery grafts. Ann Thorac Surg. 2002;73(3):779-784.

6. Bentall $\mathrm{H}$, De Bono A. A technique for complete replacement of the ascending aorta. Thorax. 1968;23(4):338-339.

7. Cabrol C, Gandjbakhch I, Cham B. Aneurysms of the ascending aorta; total replacement with reimplantation of the coronary arteries (author's transl). Nouv Presse Med. 1978;7(5):363-365.

8. Marcacci C, Trezzi M, Dreyfus GD. Recurrent Aortic Prosthetic Valve Endocarditis: A Radical Additional Anatomical Solution. Ann Thorac Surg. 2016;102(6):e577-e579. doi: 10.1016/j.athoracsur.2016.05.058

9. Prendergast BD, Tornos P. Surgery for infective endocarditis: who and when? Circulation. 2010;121(9):1141-1152. doi: 10.1161/CIRCULATIONAHA.108.773598

10. Yamazato A, Aoshima M, Nishimura N, Ban T. A case report of emergency Bentall re-operation. Kyobu Geka. 1989;42(9):778-781.

11. Hussein N, Qamar S, Abid Q. Systemic aspergilloma post aortic root surgery following coronary artery stenting: diagnostic and management dilemma. BMJ Case Rep. 2015;2015. pii: bcr2014207702. doi: 10.1136/bcr-2014-207702 